\title{
HMQ-T-F2 suppresses migration of the human cervical cancer HeLa cells by reversing EMT via the PI3K/Akt signaling pathway
}

\author{
BINGLING DAI, RUNZE YU, MENGYING FAN, TIANFENG YANG, BO WANG and YANMIN ZHANG \\ School of Pharmacy, Health Science Center, Xi'an Jiaotong University, Xi'an, Shaanxi 710061, P.R. China
}

Received January 23, 2019; Accepted May 14, 2019

DOI: $10.3892 /$ or.2019.7245

\begin{abstract}
Epithelial-mesenchymal transition (EMT) is closely related to tumor metastasis, and offers insight into novel strategies for cancer treatment. HMQ-T-F2 (F2) is a taspine derivative, which has excellent anticancer activity in human cervical cancer. The present study aimed to evaluate the effect of F2 on in vitro migration of HeLa cells. The present data demonstrated that F2 inhibited migration of HeLa cells by negatively regulating the Wnt signaling pathway and reversing EMT. F2 not only mediated Frizzled8, p-LRP6 and LRP6 expression, but also downregulated the phosphorylation of GSK3 $\beta$, and concurrently decreased the nucleus protein expression of MMP2, MMP3, MMP7, MMP9, and c-Myc. In addition, the expression of $\mathrm{N}$-cadherin, vimentin, Snail and HIF-1 $\alpha$ were downregulated and that of E-cadherin was upregulated after F2 treatment. F2 was also associated with the downregulation of the PI3K/Akt/mTOR signaling pathways. Notably, F2 induced HeLa cell accumulation at the S phase and cell apoptosis. These results provide evidence that F2 inhibits HeLa cell migration, proliferation and promotes apoptosis. It also reverses EMT, potentially via the PI3K/Akt signaling pathway. Therefore, F2 may be a potential therapeutic reagent against cervical cancer.
\end{abstract}

\section{Introduction}

Cervical cancer is one of the most common malignant tumors in females and is a serious threat to the health of females worldwide (1). In terms of incidence and death rates, cervical cancer ranks second among all gynecological malignant tumors and first among all malignant tumors in females, respectively (2). If diagnosed during the early stages, patients with cervical cancer usually have a good prognosis with a 5-year survival rate of $90 \%$ after effective treatments, like surgery, chemotherapy,

Correspondence to: Dr Yanmin Zhang, School of Pharmacy, Health Science Center, Xi'an Jiaotong University, 76 Yanta West Street, Xi'an, Shaanxi 710061, P.R. China

E-mail: zhang2008@mail.xjtu.edu.cn

Key words: HMQ-T-F2 (F2), cervical cancer HeLa cells, EMT, PI3K/Akt signaling radiotherapy, or biotherapy. However, distant metastasis of this cancer often results in poor prognosis (3). Therefore, reducing and completely preventing metastasis is key in the treatment of cervical cancer.

The metastasis of cervical cancer is a process that involves multiple factors, multiple steps, and a continuous cascade of reactions (4). In the study of cervical cancer metastasis, epithelial-mesenchymal transition (EMT) has garnered attention for being the major molecular mechanism in the metastatic cascade (5-7). EMT plays a key role in epithelial tumor progression, invasion, and metastasis. When tumor cells undergo EMT, they lose their cell polarity and cell-cell adhesion due to E-cadherin suppression and they break through the basement membrane thus obtaining mesenchymal properties such as migration and invasion (8). These cellular morphological changes enhance tumor cell migration, metastasis, and aid in the establishment of metastatic sites $(9,10)$. EMT is a dynamic and complex process that is associated with changes in multiple growth factors, protein molecules, transcription factors, as well as the respective pathways they regulate (11). Studies have reported that cadherins are one of the key components that contribute to cell motility and invasiveness via EMT (12). The reduction in E-cadherin expression and its deletion can lead to the disappearance of cell polarity and decrease cell adhesion (13). E-cadherin is a calcium-dependent transmembrane glycoprotein, which is negatively correlated with the progression, local invasion and metastasis of epithelial carcinomas. The loss of cell polarity is accompanied with an increase in markers of mesenchymal cell increase such as N-cadherin, vimentin and fibronectin. Snail is one of the transcription factors that can suppress the expression of E-cadherin. It is present in a wide range of human cancers and is associated with poor prognosis (14). Matrix metalloproteinases (MMPs) are important proteolytic enzymes, which are capable of degrading all types of extracellular matrix proteins and basement membranes (15). The EMT process leads to the initiation of metastasis $(16,17)$ and enhancement in the invasive ability of tumor cells (18). Therefore, suppression of the mesenchymal molecules associated with EMT could be an effective strategy in abolishing the EMT-triggering effect. The cervical tumor is prone to distant metastasis and has a deeper depth of tumor invasion. The PI3K/Akt pathway is a central regulator of cervical cancer and functions by regulating the cell cycle and proliferation. PI3K activation phosphorylates and activates Akt, which in turn activates the transduction of 
several downstream signals thus promoting the development of cervical cancer (19).

At present, surgery, chemotherapy, and radiotherapy are the most common treatments for cervical cancer. Development of novel natural therapeutic reagents for cervical cancer is a fast-growing field of research. According to our previous study, HMQ-T-F2, a taspine derivative, effectively inhibited cervical HeLa cell proliferation in vitro and in vivo by upregulating Axin and suppressing nuclear translocation of $\beta$-catenin (20). However, little is known about the effect of HMQ-T-F2 on HeLa cell migration. Studies have revealed that the E-cadherin-catenin complex functions in cellular adhesion and its loss has been associated with greater tumor metastasis (21). Based on these results, the effects of HMQ-T-F2 on migration of the human cervical cell line, HeLa, were further evaluated. The possible mechanism of such migration was also explored and a theoretical basis for the novel treatment of cervical cancer was provided.

\section{Materials and methods}

Materials. F2 (purity $>98 \%$ ) was designed and synthesized at the Health Science Center, Xi'an Jiaotong University. RPMI-1640, Ribonuclease (RNase), propidium iodide (PI) and Hoechst 33258 were obtained from Sigma-Aldrich; Merck KGaA. Fetal bovine serum (FBS) was purchased from HyClone; GE Healthcare Life Sciences. Trypsin was obtained from AMRESCO, Inc. Penicillin was purchased from General Pharmaceutical Factory, and streptomycin was purchased from North China Pharmaceutical. An Annexin V-FITC reagent kit was purchased from Nanjing KeyGen Biotech Co., Ltd. LRP6 rabbit mAb (cat. no. 2560S), phospho-LRP6 rabbit $\mathrm{mAb}$ (cat. no. 2568S), and phospho-GSK3 $\beta$ rabbit polyAb (Ser9) (cat. no. 5558T) were purchased from Cell Signaling Technology, Inc. MMP7 rabbit polyAb (cat. no. 10374-2-AP), MMP3 rabbit polyAb (cat. no. 17873-1-AP), c-Myc rabbit polyAb (cat. no. 10828-1-AP), Frizzled-8 rabbit polyAb (cat. no. 55093-1-AP), GSK3 $\beta$ rabbit polyAb (cat. no. 22104-1-AP), Mcl-1 rabbit polyAb (cat. no. 16225-1-AP), cyclin B1 rabbit mAb (cat. no. 55004-1-AP), cyclin D1 rabbit polyAb (cat. no. 60186-1-Ig), cyclin E rabbit polyAb (cat. no. 11554-1-AP), Bax rabbit polyAb (cat. no. 50599-2-Ig), Bcl-2 rabbit polyAb (cat. no. 12789-1-AP), MMP2 rabbit mAb (cat. no. 10373-2-AP), MMP9 rabbit $\mathrm{mAb}$ (cat. no. 10375-2-AP), anti-GAPDH (cat. no. 60004-1-Ig), E-cadherin rabbit polyAb (cat. no. 20874-I-AP), N-cadherin rabbit polyAb (cat. no. 22018-I-AP), HIF-1 $\alpha$ rabbit polyAb (cat. no. 20960-I-AP), Snail rabbit polyAb (cat. no. 26183-I-AP), phospho-Akt mouse mAb (cat. no. 66444-I-1g), Akt rabbit polyAb (cat. no. 10176-2-AP), mTOR rabbit polyAb (cat. no. 20657-I-AP) and vimentin rabbit polyAb (cat. no. A11952-1-AP) were obtained from ProteinTech Group, Inc. PI3K P110 $\alpha$ rabbit mAb (cat. no. 4249T), PI3K P110 $\beta$ rabbit $\mathrm{mAb}$ (cat. no. 3011T), PI3K P110 $\gamma$ rabbit $\mathrm{mAb}$ (cat. no. 5405T), PI3K Class III rabbit $\mathrm{mAb}$ (cat. no. 3358T), p-PI3KP85/P55 rabbit $\mathrm{mAb}$ (cat. no. 4228T), PI3KP85 rabbit $\mathrm{mAb}$ (cat. no. 4257T), phospho mTOR rabbit mAb (cat. no. 5536P), were all purchased from Cell Signaling Technology, Inc. Goat anti-rabbit IgG (cat. no. 31579), BCA protein assay reagent kit, and enhanced chemiluminescent (ECL) plus reagent kit were obtained from
Pierce; Thermo Fisher Scientific, Inc. RIPA Lysis Buffer was obtained from Applygen Technologies, Inc. Protease inhibitor cocktail, phosphatase inhibitor cocktail, and DAPI were purchased from Roche Diagnostics.

Human cell lines. Human cervical cancer cell line HeLa was purchased from Shanghai Institute of Cell Biology of the Chinese Academy of Sciences. HeLa cells were cultured in RPMI-1640 medium with $10 \%$ (v/v) FBS and incubated at $37^{\circ} \mathrm{C}$ in a $5 \% \mathrm{CO}_{2}$ atmosphere with saturated humidity.

Flow cytometric analysis of cell cycle and apoptosis. The effect of F2 on the cell cycle and apoptosis were analyzed using flow cytometry. After serum starvation, HeLa cells treated with F2 for $48 \mathrm{~h}$ were collected, washed with cold PBS and suspended in $70 \%$ ice-cold ethanol overnight at $-20^{\circ} \mathrm{C}$. Subsequently, the cells were suspended in PBS with $1 \mathrm{ml} \mathrm{RNase}(50 \mu \mathrm{g} / \mathrm{ml})$ and $1 \mathrm{ml} \mathrm{PI}(60 \mu \mathrm{g} / \mathrm{ml})$ and incubated for $30 \mathrm{~min}$ in the dark.

For cell apoptosis assay, the treated HeLa cells were harvested, washed with PBS, and sequentially stained with Annexin V-FITC. After three min, $10 \mu \mathrm{l} \mathrm{PI}(20 \mu \mathrm{g} / \mathrm{ml})$ was added to these cells and they were incubated in the dark for $10 \mathrm{~min}$.

All stained cells were analyzed using FACS (BDBiosciences). The data thus obtained were plotted using Modfit LT software 2.0 (Verity Software house).

Hoechst staining assay. HeLa cells that were treated with F2 for $48 \mathrm{~h}$, were fixed using $4 \%$ paraformaldehyde for $10 \mathrm{~min}$. After washing with PBS, cells were stained with Hoechst 33258 in the dark for $20 \mathrm{~min}$. The cells were photographed using an inverted fluorescence microscope (DM505; Nikon Corp.).

Wound scratch assay. The wound scratch assay was performed in order to evaluate directional cell migration. Briefly, when HeLa cells grew to $70-80 \%$ confluence, cell monolayers were wounded to form a scratch using sterile pipette tips (100-200 $\mu$ l). After washing with PBS to remove cell debris, cells were incubated in either the absence or presence of F2 in RPMI-1640 medium with 5\% FBS. The cells were photographed at the beginning $(0 \mathrm{~h})$ and then at 24 and $48 \mathrm{~h}$. The migration distance was measured using an image analysis software (NIS-Elements Viewer 4.2.0; Nikon Corporation) and the migration rate was calculated.

Transwell migration assay. The HeLa cells were seeded onto a Transwell chamber and incubated with F2 for $48 \mathrm{~h}$. Then the medium in the chamber was replaced with serum-free medium and the lower chamber was filled with RPMI-1640 medium containing $30 \% \mathrm{FBS}$ as a chemoattractant. After culturing for $24 \mathrm{~h}$, the cells that did not migrate and remained at the top of the chamber were removed carefully using a cotton swab. The migrating cells, that settled at the bottom of the chamber were fixed using methanol and stained using $0.2 \%$ crystal violet for $15 \mathrm{~min}$. The cells that had migrated were photographed and counted based on 5-field digital images obtained randomly at a magnification of $\mathrm{x} 100$.

Western blot analysis. Total protein was lysed using ice-cold RIPA with a protease inhibitor cocktail and a phosphorylated 
A
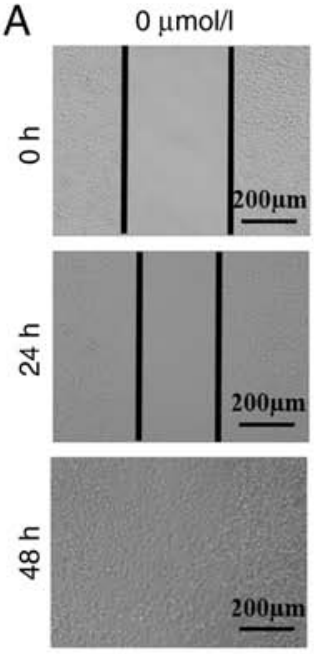

C

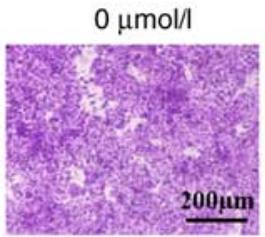

$0.15 \mu \mathrm{mol} / \mathrm{l}$
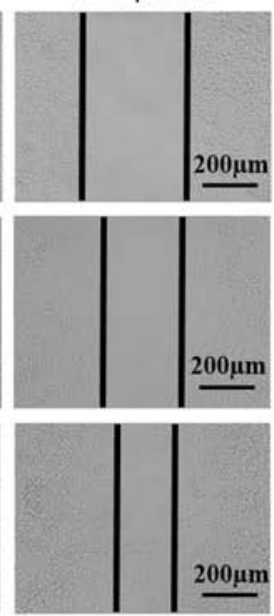

$0.15 \mu \mathrm{mol} / \mathrm{l}$

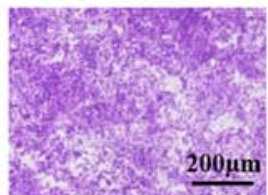

$0.3 \mu \mathrm{mol} / \mathrm{l}$
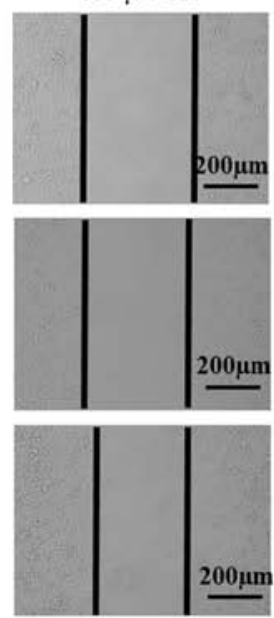

$0.3 \mu \mathrm{mol} / \mathrm{l}$

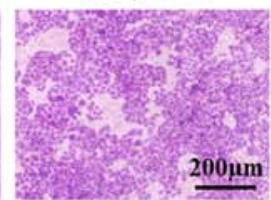

$0.6 \mu \mathrm{mol} / \mathrm{I}$
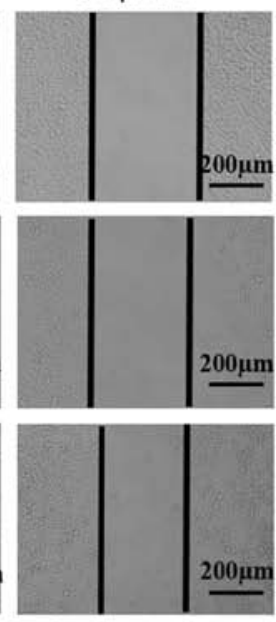

$0.6 \mu \mathrm{mol} / \mathrm{l}$

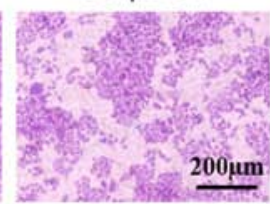

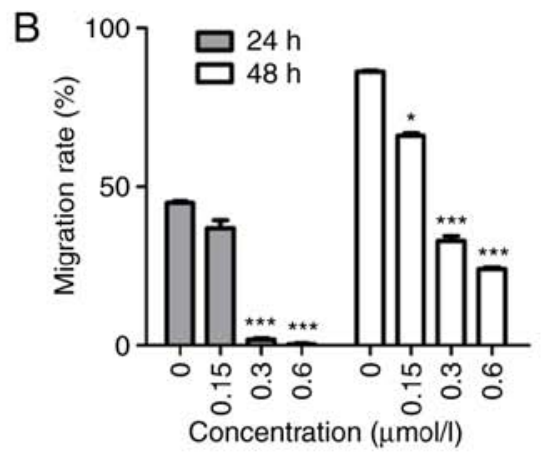

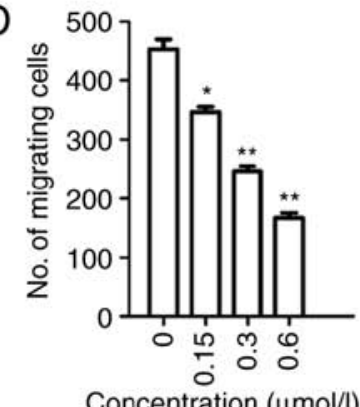

Figure 1. Effect of F2 on migration of HeLa cell lines. Cells were treated with vehicle control or the indicated concentrations of F2. (A) The migration ability of HeLa cells was evaluated in each group using a wound healing assay at 24 and $48 \mathrm{~h}$. (B) Quantification of migrated cells in A. (C) The ability of HeLa cells to invade was detected using a Transwell migration assay. HeLa cells migrated through the polycarbonate membrane stained with $0.2 \%$ crystal violet. (D) Quantification of migrated cells in C. Values are presented as the means $\pm \operatorname{SEM}(\mathrm{n}=3)$. ${ }^{*} \mathrm{P}<0.05,{ }^{* * *} \mathrm{P}<0.01,{ }^{* * * *} \mathrm{P}<0.001$ vs. the control group $(0 \mu$ mol/l). HMQ-T-F2, F2.

protease inhibitor cocktail and was used for HeLa cells that were treated with $\mathrm{F} 2$ for $48 \mathrm{~h}$. After quantification using a BCA assay, that was performed in accordance with the manufacturer's instructions, the protein sample $30 \mu \mathrm{g}$ was separated by the $10 \%$ SDS-PAGE and transferred to a PVDF membrane. Subsequently, the membranes were blocked using $5 \%$ BSA for $2 \mathrm{~h}$ at room temperature and incubated with the indicated primary antibodies that were diluted by $1 \mathrm{X}$ TBST buffer overnight at $4^{\circ} \mathrm{C}$. After washing, the membranes were incubated with species-specific horseradish peroxidase (HRP)-conjugated secondary antibodies for $1 \mathrm{~h}$ at $37^{\circ} \mathrm{C}$ and the resultant antigen-antibody complexes were visualized using an enhanced chemiluminescence (ECL) kit. The images were scanned using chemiluminescent and fluorescent imaging systems (Champchemi ${ }^{\mathrm{TM}}$ Professional; cat. no. SG2010084; Beijing Sage Creation Science Co., Ltd.) and the bands were quantified using Image-Pro plus software (Image-Pro Plus 5.1; Media Cybernetics, Inc.). GAPDH was used as an internal control.

Statistical analysis. One-way analysis of variance (ANOVA) with Tukey's multiple comparison test or Student's unpaired t-test was used to analyze significance using SPSS 19.0 (IBM Corp.) and GraphPad Prism 5 software (GraphPad Software). A P-value $<0.05$ was considered to indicate a statistically significant difference. ${ }^{*} \mathrm{P}<0.05,{ }^{* *} \mathrm{P}<0.01$ and ${ }^{* * *} \mathrm{P}<0.001$ vs. the control group. Data are expressed as the means \pm SEM.

\section{Results}

F2 inhibits cervical cancer HeLa cell migration. It is known that cell migration is important for the progression of malignant tumors. The ability of F2 to inhibit migration was evaluated using wound scratch and Transwell migration assays. Our previous study concluded that treatment of F2 at a dosage of $0.6 \mu \mathrm{mol} / 1$ for $48 \mathrm{~h}$ had no cytotoxic effect on HeLa cells (20). In addition, an MTT assay revealed that F2 had no inhibition on human normal cervical epithelial cells even at $25 \mu \mathrm{mol} / \mathrm{l}$, in the previous study. Hence, F2 was used at a concentration of $0.6 \mu \mathrm{mol} / 1$ for the experiments and 5\% FBS was used to exclude the anti-proliferation effect of $\mathrm{F} 2$ in the migration assay for $48 \mathrm{~h}$. As revealed in Fig. 1A and B, HeLa cells migrated to fill the scratched area after $48 \mathrm{~h}$ in the absence of F2, while the migration rate decreased in a dose-dependent manner upon F2 treatment at 24 and $48 \mathrm{~h}$. According to the Transwell assay results, the number of HeLa cells migrating through the chamber significantly decreased in the group that received F2 treatment, when compared to the control group (Fig. 1C and D). Both these assays confirmed that F2 inhibited HeLa cell migration.

F2 inhibits Wnt signaling proteins in HeLa cells. $\beta$-catenin is an important molecule in the Wnt signaling pathway. In our previous study, it was revealed that $\mathrm{F} 2$ arrests the translocation of $\beta$-catenin to the nucleus in a dose-dependent manner. Thus, 

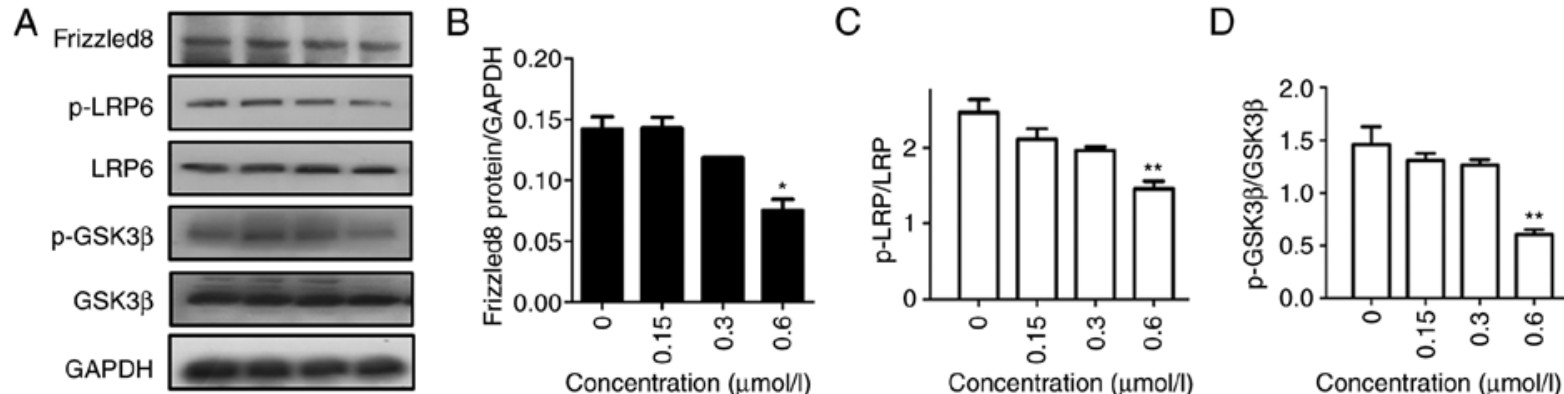

Figure 2. Effect of F2 on the Wnt signaling pathway proteins in HeLa cells. (A) Western blot analysis of cell membrane proteins, including Frizzled-8, p-LRP, LRP, p-GSK $3 \beta$ and GSK3 $\beta$ in the Wnt signaling pathway of HeLa cells. The cells were quantified using relative densitometry values normalized to GAPDH. (B-D) Quantification of A. Values are presented as the means \pm SEM. ${ }^{*} \mathrm{P}<0.05,{ }^{* *} \mathrm{P}<0.01$ vs. the untreated control cells. HMQ-T-F2, F2; p-LRP, phosphorylated LRP; GSK3 $\beta$, phosphorylated GSK3 $\beta$.

A

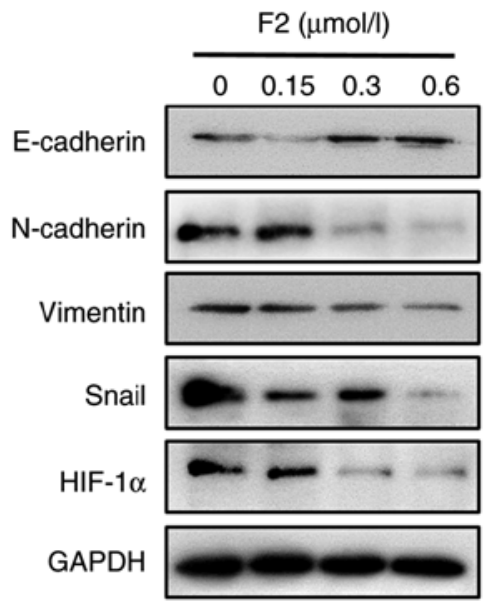

B

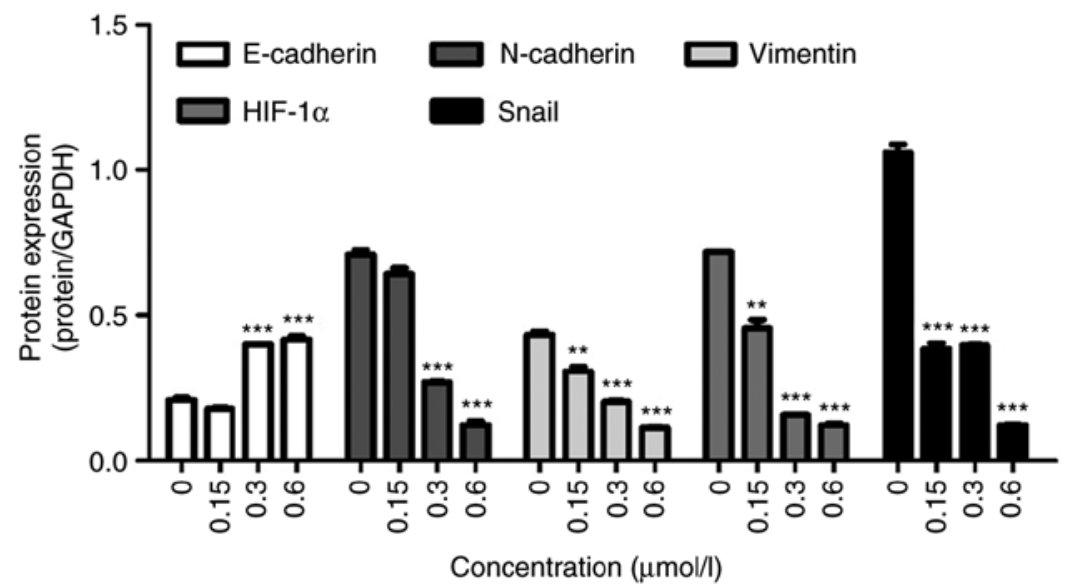

Figure 3. Effect of F2 on the EMT signaling pathway-related proteins in HeLa cells. (A) Western blot analysis of EMT signaling pathway-related proteins, including E-cadherin, N-cadherin, vimentin, Snail and HIF-1 $\alpha$. (B) Quantification of A. Values are presented as the means \pm SEM ( $=3$ ). ${ }^{* *} \mathrm{P}<0.01$, ${ }_{* * * *} \mathrm{P}<0.001$ vs. the control group $(0 \mu \mathrm{mol} / \mathrm{l})$. HMQ-T-F2, F2; EMT, epithelial-mesenchymal transition.

the expression of Wnt signaling proteins was investigated after exposure to $\mathrm{F} 2$ for $48 \mathrm{~h}$ in order to determine whether inhibition of migration by $\mathrm{F} 2$ was associated with Wnt signaling. The data revealed that the expression of Frizzled-8, phosphorylation of LRP-5/6, and phosphorylation of GSK3 $\beta$ decreased in a concentration-dependent manner (Fig. 2), indicating that F2 inhibited the migration via the Wnt signaling pathway.

F2 inhibits EMT-related molecules in HeLa cells. Induction of EMT is the initial process by which epithelial cancer cells acquire motility, thus promoting migration and invasion (22). A western blot assay was performed in order to determine the effect of F2 on the expression of EMT-related proteins. As revealed in Fig. 3, F2 significantly increased the expression of E-cadherin, and decreased the expression of $\mathrm{N}$-cadherin, vimentin and Snail. These results indicated that F2 can inhibit the migration of HeLa cells by reversing EMT.

HIF-1 $\alpha$ plays an important role in tumor cell metastasis and cancer function (23). Using a western blot assay, it was revealed that F2 decreased HIF-1 $\alpha$ expression (Fig. 3). HIF-1 $\alpha$ promotes tumor metastasis by increasing the expression of MMPs that play an important role in metastatic foci formation (23). Western blot analysis demonstrated that the expression of MMP2, MMP3, MMP7, and MMP9 was downregulated in HeLa cells treated with F2 (Fig. 4). In addition, c-Myc protein expression decreased after F2 treatment (Fig. 4). MMPs and $\mathrm{c}-\mathrm{Myc}$ are the nuclear targets of the $\mathrm{Wnt} / \beta$-catenin signaling pathway. These observations were consistent with our previous study, which reported that F2 markedly inhibited HeLa cells by targeting $\beta$-catenin.

F2 impairs the PI3K/Akt signaling pathway. The PI3K/Akt pathway is involved in the regulation of HIF-1 $\alpha$ and is closely related to cell proliferation. Western blot analysis revealed that F2 inhibited the PI3K/Akt signaling pathway in a dose-dependent manner along with the inhibition of HIF-1 $\alpha$ expression. As revealed in Fig. 5, F2 inhibited the PI3Kp110 $\alpha$ subunit and the phosphorylation of PI3Kp85/p55, Akt and mTOR, which are important molecules in PI3K signaling.

F2 induces the accumulation of HeLa cells in the S phase. PI staining and flow cytometry were used to analyze the cell cycle distribution in the HeLa cells following treatment with F2. The results revealed that F2 significantly arrested the HeLa cells in their $\mathrm{S}$ phase. The HeLa cells were treated with $0,0.15,0.3$ and $0.6 \mu \mathrm{mol} / \mathrm{l}$ of $\mathrm{F} 2$ for $48 \mathrm{~h}$, and the percentage of cells in the 

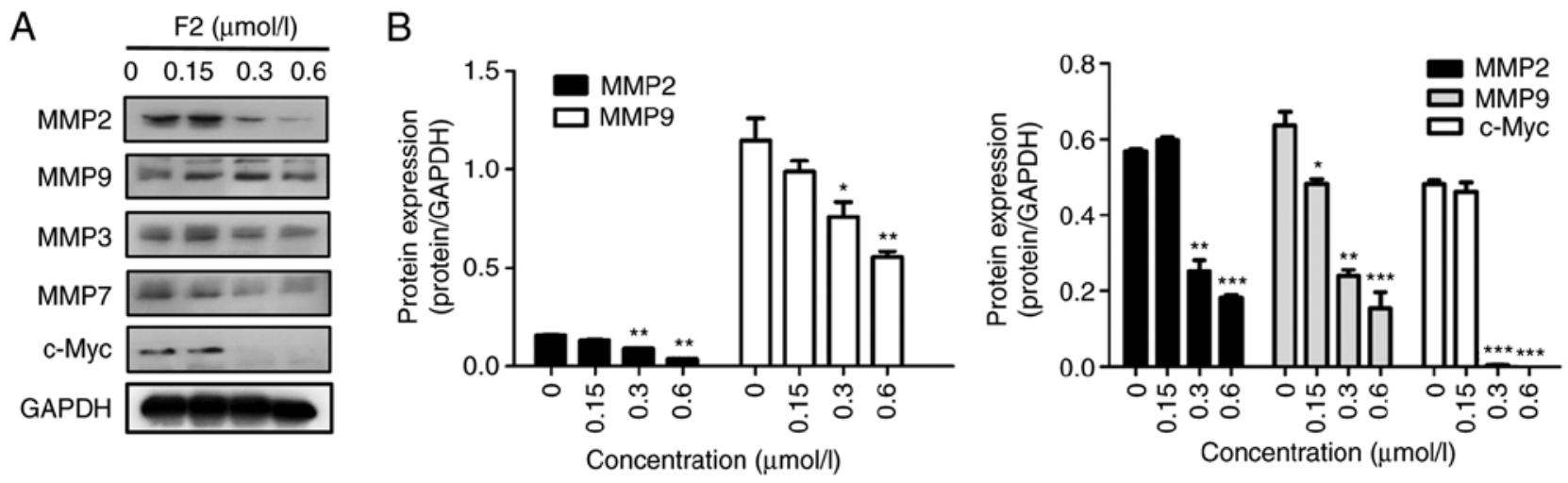

Figure 4. Effect of F2 on the Wnt signaling pathway proteins in HeLa cells. (A) Western blot analysis of cell nucleus proteins, including MMP2, MMP9, MMP3, MMP7 and c-Myc in the Wnt signaling pathway of HeLa cells. (B) Quantification of A. The results presented are representative of three independent experiments. Data are expressed as the mean \pm SEM. ${ }^{*} \mathrm{P}<0.05,{ }^{* *} \mathrm{P}<0.01,{ }^{* * *} \mathrm{P}<0.001$ vs. the control group $(0 \mu$ mol/1). HMQ-T-F2, F2; MMP, matrix metalloproteinase.

A

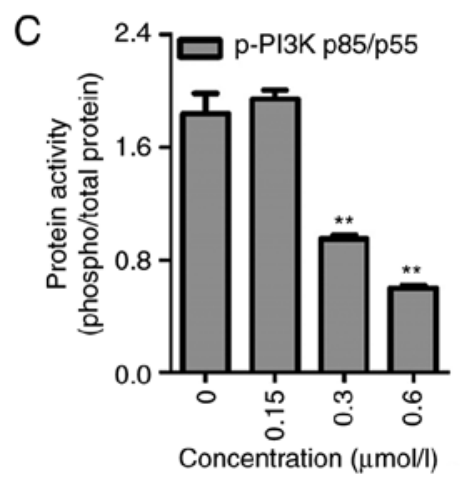

B

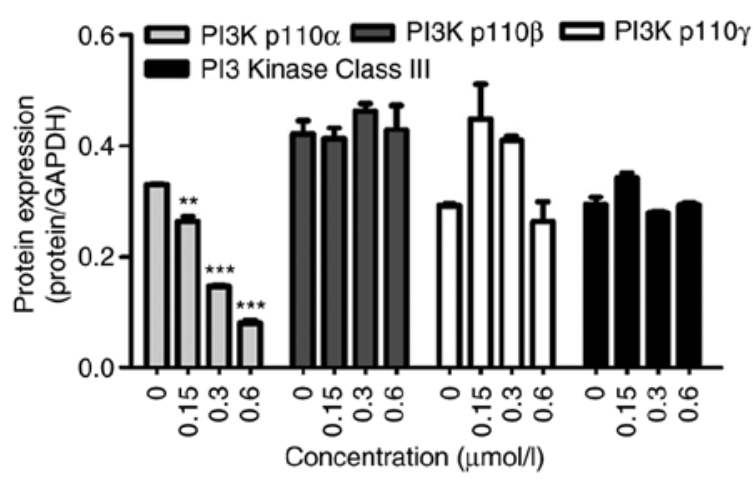

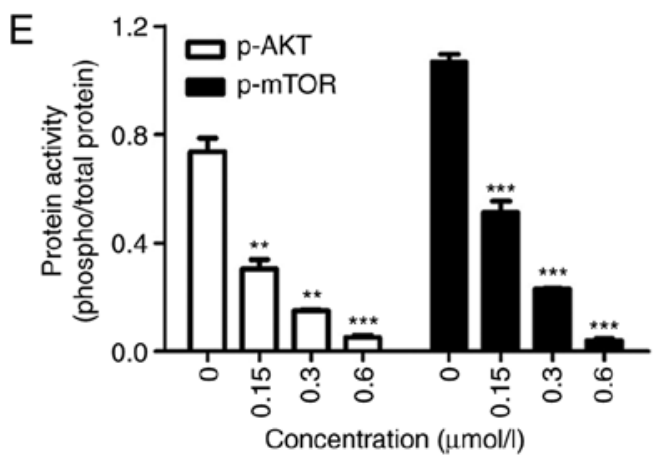

Figure 5. Effect of F2 on the PI3K/Akt signaling pathway proteins in HeLa cells. (A) Western blot analysis of PI3K-subtype proteins, including PI3K p110 $\alpha$, PI3K p110 $\beta$, PI3K p110 $\gamma$, PI3K Class III and p-PI3K p86/p55. (B and C) Quantification of A. (D) Effect of F2 on phosphorylation of Akt and mTOR. (E) Quantification of D. Values are presented as the means $\pm \operatorname{SEM}(\mathrm{n}=3) .{ }^{* *} \mathrm{P}<0.01,{ }^{* * *} \mathrm{P}<0.001$ vs. the control group $(0 \mu \mathrm{mol} / 1)$. HMQ-T-F2, F2.

S phase increased from $38.07 \%$ in vehicle controls to $40.76,48.96$ and $62.38 \%$, respectively. Conversely, the percentage of cells in the G1 phase decreased from 51.47 to $50.7,45.72$ and $31.85 \%$, while the percentage of cells in the $\mathrm{G} 2 / \mathrm{M}$ phase decreased from 10.47 to $8.55,5.34$ and $5.78 \%$, respectively (Fig. 6A and B).

To explore the mechanisms underlying F2-mediated $\mathrm{S}$ phase arrest, the effect of F2 on key cell cycle-related proteins was examined. It is well known that cyclin $\mathrm{E}$ is closely correlated with $\mathrm{S}$ phase arrest, and the present results revealed that F2 upregulated the expression of cyclin E (Fig. 6C and D). In addition, treatment with $\mathrm{F} 2$ also downregulated the expres- sion of cyclin D1 and cyclin B1, which are closely related to the $\mathrm{G} 1$ and $\mathrm{G} 2 / \mathrm{M}$ phase, respectively. Thus, the data revealed that F2 induced HeLa cell cycle arrest at the $S$ phase.

F2 induces HeLa cell apoptosis and regulates cell apoptosis-regulatory molecules. Annexin V-PI staining and flow cytometric analysis were used to determine whether F2 inhibits HeLa cell proliferation by inducing cell apoptosis. As revealed in Fig. 7A, the FACS results revealed that the percentage of apoptotic cells (the sum of lower right quadrant and upper right quadrant) in the vehicle group was $12.89 \%$, and that the 

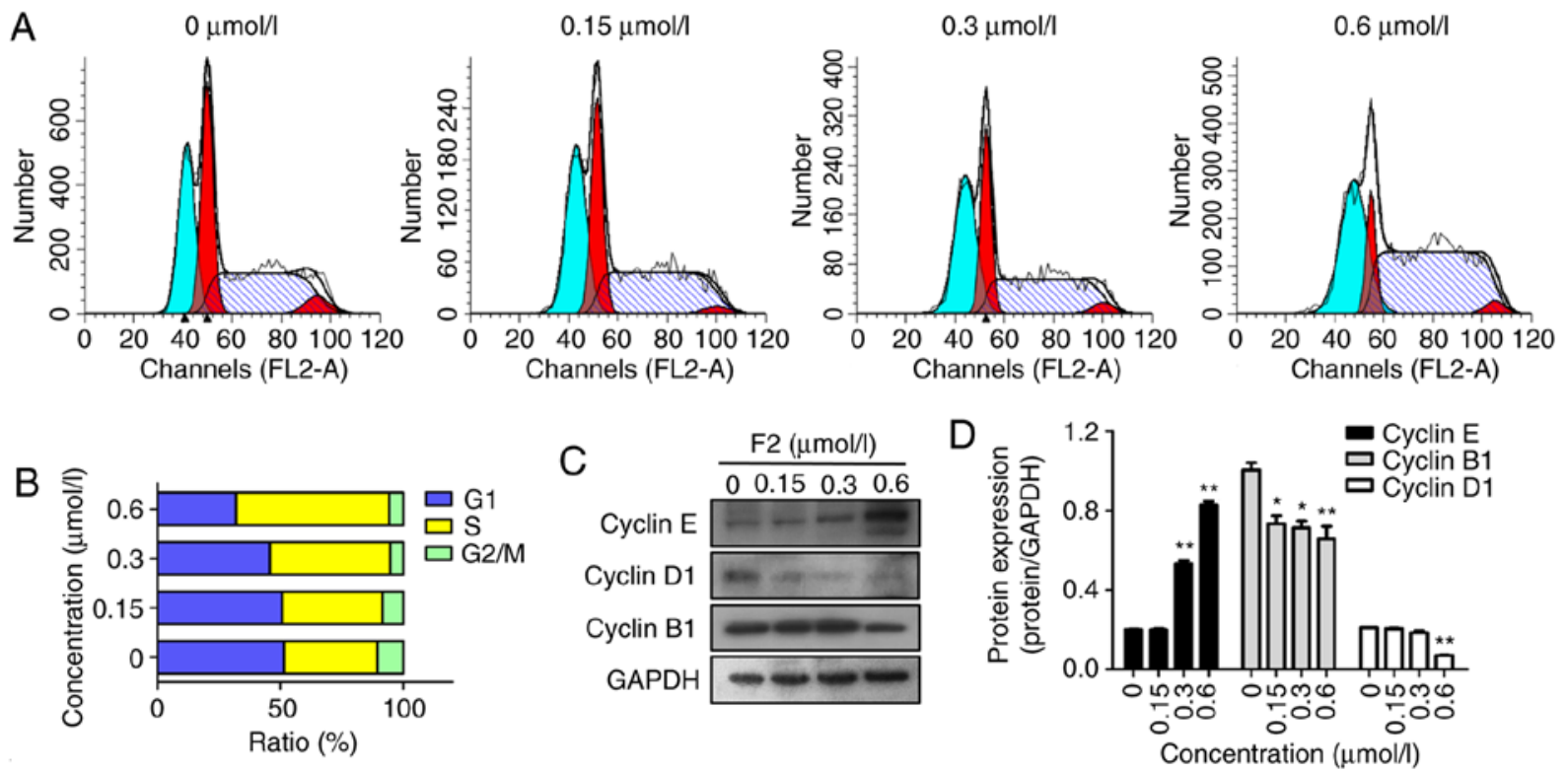

Figure 6. F2 arrests HeLa cells in the S phase and regulates cyclin protein levels. (A) Effect of F2 on HeLa cell cycle progression. HeLa cells were treated with $\mathrm{F} 2$ for $48 \mathrm{~h}$ and stained with PI. DNA content was then assessed using flow cytometry. (B) Quantitation of the data in A. (C) Effect of F2 on the expression of cell-cycle proteins, cyclin E, cyclin D1 and cyclin B1, was studied using western blot analysis. (D) Results were quantified using densitometry analysis of the bands from $\mathrm{C}$. Values are presented as the means \pm SEM $(\mathrm{n}=3)$. ${ }^{*} \mathrm{P}<0.05,{ }^{* *} \mathrm{P}<0.01$ vs. the control group $(0 \mu \mathrm{mol} / \mathrm{l})$. HMQ-T-F2, F2.

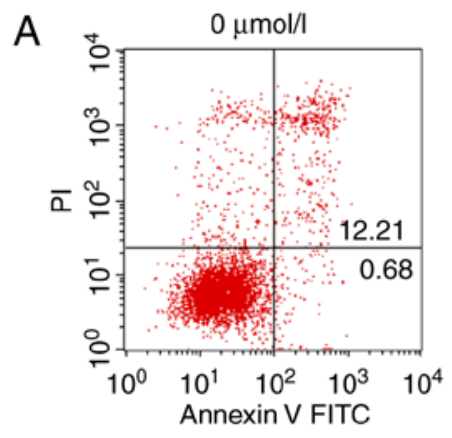

B

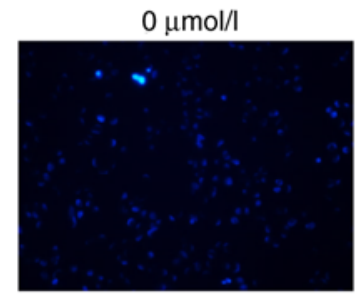

C

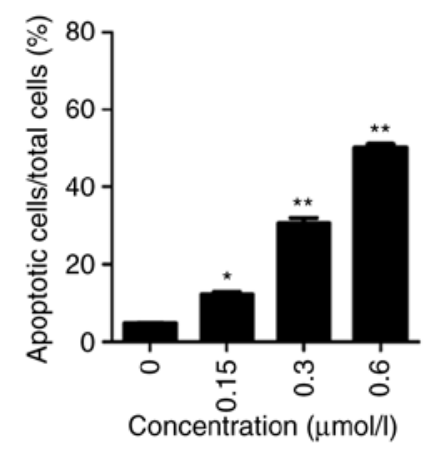

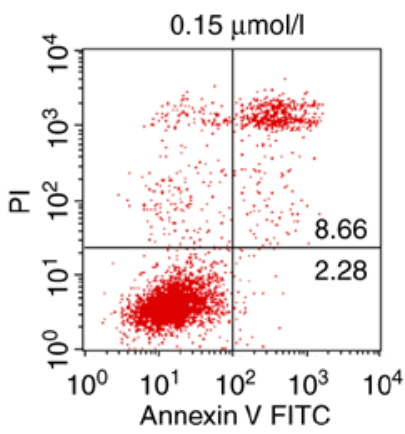
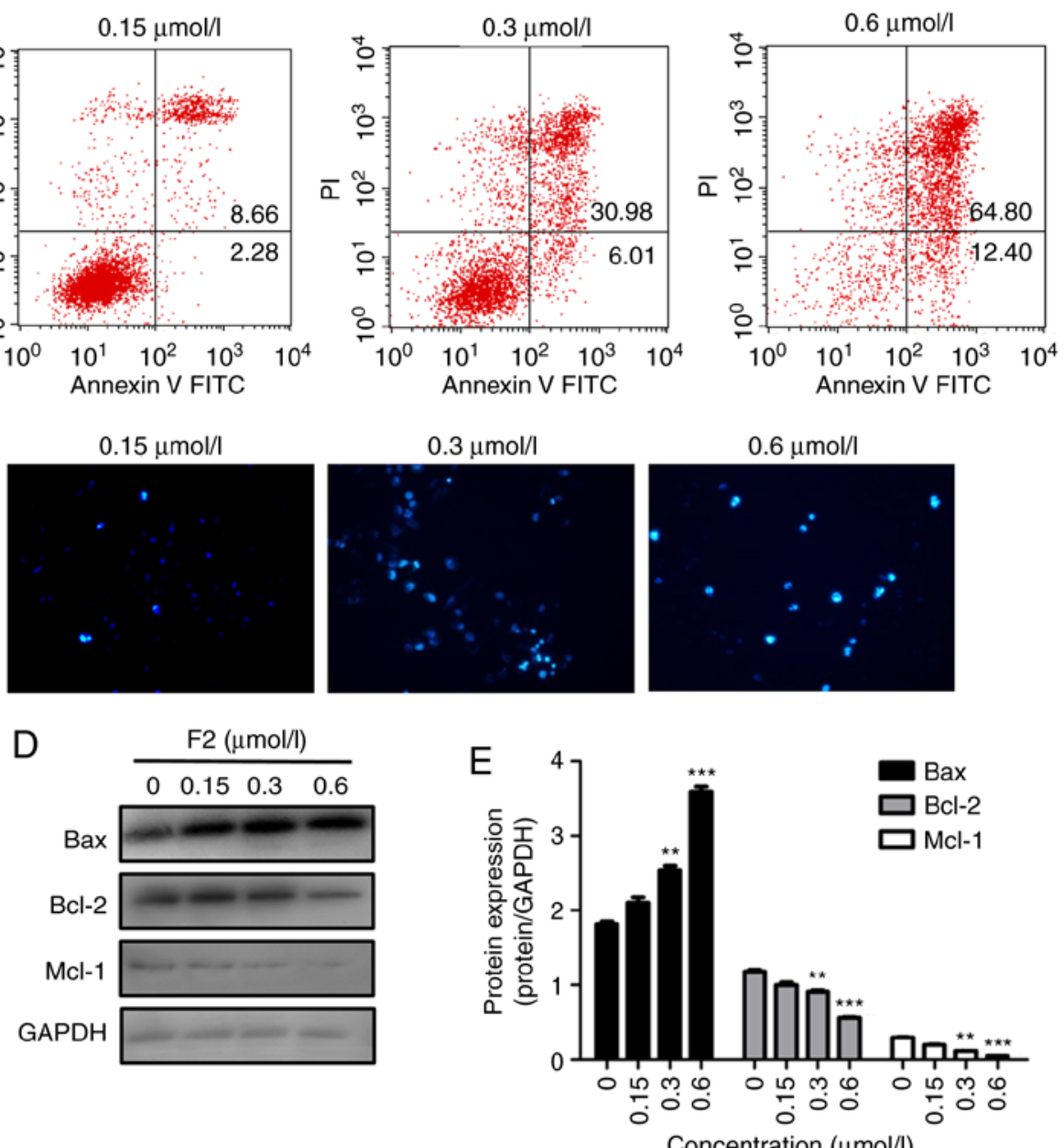
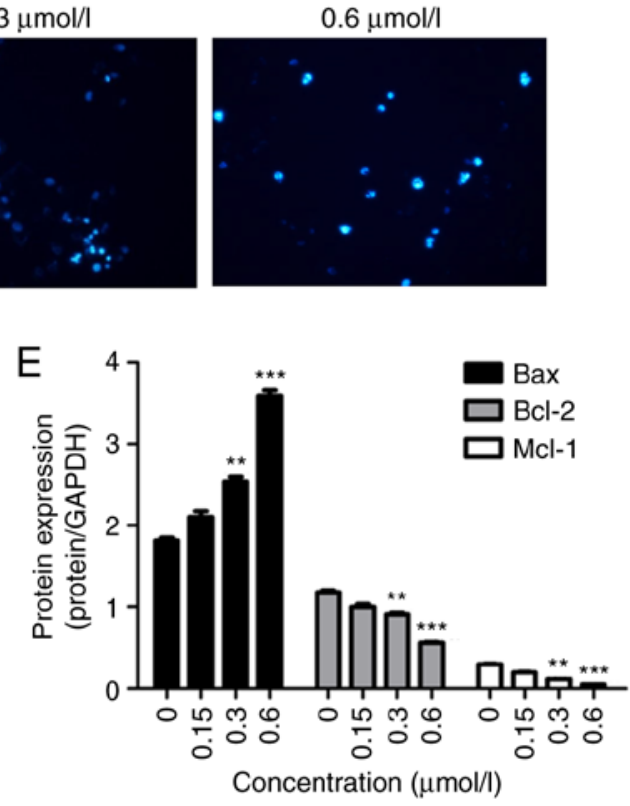

Figure 7. F2 induces HeLa cell apoptosis and regulates levels of cell apoptosis proteins. (A) Cell apoptosis was measured using flow cytometric analysis of Annexin V-FITC/PI-stained HeLa cells treated with F2. The percentage of apoptotic cells is indicated. (B) The morphology of apoptotic nuclei was observed after treatment with F2 using Hoechst 33258 staining. (C) Quantification of apoptotic cells/total cells in B. (D) Western blot analysis of Bax, Bcl-2 and Mcl-1 expression in HeLa cells treated with F2. (E) Results were quantified using densitometry analysis of the bands from D. Values are presented as the means \pm SEM $(\mathrm{n}=3) .{ }^{* * *} \mathrm{P}<0.01,{ }^{* * *} \mathrm{P}<0.001$ vs. the control group $(0 \mu \mathrm{mol} / \mathrm{l})$. HMQ-T-F2, F2. 
percentage of apoptotic cells increased after treatment with F2. The percentage of apoptotic cells in F2-treated groups were 10.94, 36.99 and 77.20, respectively. In addition, Hoechst staining was applied to observe the morphology of HeLa cells. The results indicated that F2 induced the condensed bright blue apoptotic nuclei in HeLa cells (Fig. 7B and C). Induction of apoptosis was further confirmed by upregulation of the pre-apoptotic protein Bax and downregulation of the anti-apoptotic proteins, Bcl-2 and Mcl-1 (Fig. 7D and E). The data indicated that F2 activity was related to programmed cell death.

\section{Discussion}

Every year there are $>500,000$ new cases of cervical cancer and 200,000 cervical cancer-related deaths worldwide, with the majority of these occurring in developing countries (24). Although existing cervical cancer screening techniques and HPV have improved the rate at which this disease is diagnosed to some extent, reoccurrence and metastasis still result in poor outcomes $(25,26)$. Therefore, novel and efficient therapeutic targets for cancer metastasis are required.

Our previous study demonstrated that $\beta$-catenin is an important target of $\mathrm{F} 2$, and it was confirmed that $\mathrm{F} 2$ regulates the $\beta$-catenin destruction complex that consists of Axin, APC, and CK1 (20). It is well known that $\beta$-catenin is a key player in abnormal Wnt signaling and is the most important oncogene in cervical cancer biology due to its ability to regulate cell migration. In order to elucidate the mechanism of inhibition of HeLa cell migration by F2, wound healing and Transwell assays were used. The results revealed that $\mathrm{F} 2$ could inhibit the migration of HeLa cells. The activation of Wnt $/ \beta$-catenin signaling in cancers mostly occurs as a result of the binding of the Wnt ligand with its receptor LRP5/6 and Frizzled8. It also presents the inactivation of GSK-3 $\beta$, which leads to the increase in the activity and quantitative stabilization of $\beta$-catenin. The present results revealed that F2 suppressed phosphorylation of LRP5/6 and expression of Frizzled8. LRP5/6 and Frizzled8 are transmembrane receptors of the Wnt/Frizzled8 pathway. In addition, the inhibition of F2 on phosphorylated LRP5/6 resulted in the suppression of the phosphorylation of GSK $3 \beta$.

Furthermore, several studies have confirmed that the Wnt signaling pathways regulate the process of EMT in cancer. The key factor of the Wnt signaling pathway, $\beta$-catenin, induces EMT by increasing E-cadherin suppressors $(27,28)$. EMT is reported to promote the invasive ability and metastasis of cervical cancer and is positively associated with poor prognosis (29). When Wnt signaling is activated, the number of EMT target proteins increase, and vice versa. These findings reveal that the crosstalk between Wnt/ $\beta$-catenin signaling and the EMT process forms a positive feedback loop (30). Therefore, when EMT-related proteins were evaluated using western blotting, the results revealed that F2 inhibited the expression of Snail. In addition, F2 also significantly upregulated the expression of epithelial marker E-cadherin, and downregulated the expression of interstitial markers $\mathrm{N}$-cadherin and vimentin. These results indicated that F2 can inhibit the migration of HeLa cells by reversing EMT. The overexpression of HIF-1 $\alpha$ protein has been reported in several human cancers including cervical, breast, and ovarian cancer (31-33). Clinically, high levels of HIF-1 $\alpha$ have several functions in cancer biology including angiogenesis, cell survival, tumor metastases and overcoming hypoxia (23). Using western blot analysis, it was revealed that F2 decreased HIF-1 $\alpha$ expression. HIF-1 $\alpha$ promotes the metastasis of malignant tumors by increasing the levels of MMPs (34). MMPs are also known to play a major role in different cell behaviors such as cell proliferation, migration, infiltration, differentiation, angiogenesis and metastatic foci formation (15). Western blot analysis revealed that the expression of MMP2, MMP3, MMP7, MMP9 and c-Myc were downregulated in HeLa cells treated with F2. In addition, MMPs and c-Myc are the nuclear targets of the Wnt/ $\beta$-catenin signaling pathway. These observations were consistent with our previous study that revealed that F2 significantly inhibited HeLa cells by targeting $\beta$-catenin. The $\mathrm{PI} 3 \mathrm{~K} / \mathrm{Akt} / \mathrm{mTOR}$ signaling pathway is involved in the development and progression of tumors. It is aberrantly activated in cervical cancer and is closely related to tumor recurrence and metastasis (35). PI3K/Akt/mTOR signaling is also an intracellular signaling pathway that regulates the cell cycle and can induce the occurrence of EMT (36). In the present study, it was revealed that F2 inhibited the phosphorylation of PI3Kp85/p55 and its downstream signaling molecules, Akt and mTOR. Thus, the results of our present and previous studies, collectively, revealed that $\mathrm{F} 2$ inhibited the migration and proliferation of HeLa cells by reversing EMT, and negatively regulating the Wnt/ $\beta$-catenin and PI3K/Akt signaling pathways.

In addition, the present research results revealed that F2 arrested the HeLa cell cycle at the S phase. Certain key proteins involved in regulating cell cycle transition were also studied. The results revealed that F2 induced HeLa cell arrest and was also associated with the downregulation of cyclin D1 and cyclin $\mathrm{B} 1$ and upregulation of cyclin $\mathrm{E}$. The results also revealed that F2 induced HeLa cell apoptosis by downregulating Bcl-2 and Mcl-1 and upregulating Bax. These data partly reflect the ability of F2 to inhibit HeLa cell proliferation. Nevertheless, further studies are needed on the mechanism of whcih F2 suppresses migration of the human cervical cancer HeLa cells and the druggability of F2.

In conclusion, the present findings demonstrated that F2 can impair migration and proliferation of the human cervical cancer HeLa cells. F2 induced HeLa cell arrest at the S phase and apoptosis. F2 reversed EMT, thus potentially inhibiting both the Wnt/ $\beta$-catenin and PI3K/Akt signaling pathways, and downregulating HIF-1 $\alpha$ expression.

\section{Acknowledgements}

Not applicable.

\section{Funding}

The present study was supported by the National Natural Science Foundation of China (grant nos. 81503101 and 81773772), the Fundamental Research Funds for the Central Universities (xjj2018167) and the National Science Foundation for Post-Doctoral Scientists of China (grant no. 2019M653670).

\section{Availability of data and materials}

The datasets used during the present study are available from the corresponding author upon reasonable request. 


\section{Authors' contributions}

BD was responsible for collection of data, data analysis and interpretation, and manuscript writing. RY and MF carried out the experiments. TY and BW analyzed the data and reviewed the manuscript. BD and $\mathrm{YZ}$ was responsible for the conception and design of the study, and financial support. All authors read and approved the final manuscript and agree to be accountable for all aspects of the research in ensuring that the accuracy or integrity of any part of the work are appropriately investigated and resolved.

\section{Ethics approval and consent to participate}

Not applicable.

\section{Patient consent for publication}

Not applicable.

\section{Competing interests}

The authors declare that they have no competing interests.

\section{References}

1. MarthC,LandoniF,MahnerS,McCormackM,Gonzalez-Martin A and Colombo N; ESMO Guidelines Committee: Cervical cancer: ESMO clinical practice guidelines for diagnosis, treatment and follow-up. Ann Oncol 28 (Suppl): iv72-iv83, 2017.

2. Fomenko Y, Cialkowska-Rysz A, Muravlyova L, Sirota V and Sapar B: Assessment of direct results of cervical cancer combined treatment. Georgian Med News: 21-24, 2018.

3. Zhang L, Qian H, Sha M, Luan Z, Lin M, Yuan D, Li X, Huang J and Ye L: Downregulation of HOTAIR expression mediated anti-metastatic effect of artesunate on cervical cancer by inhibiting COX-2 expression. PLoS One 11: e0164838, 2016.

4. Chu SC, Yu CC, Hsu LS, Chen KS, Su MY and Chen PN Berberine reverses epithelial-tomesenchymal transition and inhibits metastasis and tumor-induced angiogenesis in human cervical cancer cells. Mol Pharmacol 86: 609-623, 2014.

5. Lee JM, Dedhar S, Kalluri R and Thompson EW: The epithelial-mesenchymal transition: New insights in signaling, development, and disease. J Cell Biol 172: 973-981, 2006.

6. Wang Z, He S, Guo P, Guo X and Zheng J: Microrna-1297 inhibits metastasis and epithelial-mesenchymal transition by targeting aeg-1 in cervical cancer. Oncol Rep 38: 3121-3129, 2017.

7. Sathyanarayanan A, Chandrasekaran KS and Karunagaran D: Microrna-145 modulates epithelial-mesenchymal transition and suppresses proliferation, migration and invasion by targeting sip1 in human cervical cancer cells. Cell Oncol (Dordr) 40: 119-131, 2017.

8. Qi X,Zhang L and Lu X: New insights into the epithelial-to-mesenchymal transition in cancer. Trend Pharmacol Sci 37: 246-248, 2016.

9. Franco-Chuaire ML, Magda Carolina SC and Chuaire-Noack L: Epithelial mesenchymal transition (EMT): Principles and clinical impact in cancer therapy. Invest Clin 54: 186-205, 2013.

10. Wang Y, Wen M, Kwon Y, Xu Y, Liu Y, Zhang P, He X, Wang Q, Huang Y, Jen KY, et al: CUL4A induces epithelial-mesenchymal transition and promotes cancer metastasis by regulating ZEB1 expression. Cancer Res 74: 520-531, 2014.

11. Li HQ and Ke Y: Mechanism of epithelial-mesenchymal transition. Chin Pharmacol Bull 33: 1342-1344, 2017.

12. Wheelock MJ, Shintani Y, Maeda M, Fukumoto Y and Johnson KR: Cadherin switching. J Cell Sci 121: 727-735, 2008.

13. Gos M, Miloszewska J and Przybyszewska M: Epithelialmesenchymal transition in cancer progression. Postepy Biochem 55 121-128, 2009 (In Polish).

14. Sethi S, Macoska J, Chen W and Sarkar FH: Molecular signature of epithelialmesenchymal transition (EMT) in human prostate cancer bone metastasis. Am J Transl Res 3: 90-99, 2010.
15. Li W, Li S, Deng L, Yang S, Li M, Long S, Chen S, Lin F and Xiao L: Decreased MT1-MMP in gastric cancer suppressed cell migration and invasion via regulating MMPs and EMT. Tumor Biol 36: 6883-6889, 2015.

16. Hugo HJ, Kokkinos MI, Blick T, Ackland ML, Thompson EW and Newgreen DF: Defining the E-cadherin repressor interactome in epithelial mesenchymal transition: The PM C42 model as a case study. Cells Tissues Organs 193: 23-40, 2011.

17. Nguyen PT, Kudo Y, Yoshida M, Iizuka S, Ogawa I and Takata T: $\mathrm{N}$-cadherin expression is correlated with metastasis of spindle cell carcinoma of head and neck region. J Oral Pathol Med 40: 77-82, 2011

18. Wu Y and Zhou BP: TNF-alpha/NF-kappaB/Snail pathway in cancer cell migration and invasion. Br J Cancer 102: 639-644, 2010.

19. Wang YL, Liu HF, Shi XJ and Wang Y: Antiproliferative activity of Farnesol in HeLa cervical cancer cells is mediated via apoptosis induction, loss of mitochondrial membrane protential and PI3K/Akt signaling pathway. J Buon 23: 752-757, 2018

20. Dai B, Yang T, Ma Y, Ma N, Shi X, Zhang D, Zhang J and Zhang Y: HMQ-T-F2 exert antitumour effects by upregulation of Axin in human cervical HeLa cells. J Cell Mol Med 22: 2955-2959, 2018.

21. Beavon IR: The E-cadherin-catenin complex in tumour metastasis: Structure, function and regulation. Eur J Cancer 36: 1607-1620, 2000.

22. Bahnson A, Athanassiou C, Koebler D, Qian L, Shun T, Shields D, Yu H, Wang H, Goff J, Cheng T, et al: Automated measurement of cell motility and proliferation. BMC Cell Biol 6: 19,2005.

23. Semenza GL: Targeting HIF-1 for cancer therapy. Nat Rev Cancer 10: 721-732, 2003 .

24. Siegel R, Ma J, Zou Z and Jemal A: Cancer statistics, 2014. CA Cancer J Clin 64: 9-29, 2014.

25. Yan J, Zhang Y, Ren C, Shi W and Chen L: Involvement of nuclear protein $\mathrm{C} 23$ in activation of EGFR signaling in cervical cancer. Tumour Biol 37: 905-910, 2016.

26. Yee GP, de Souza P and Khachigian LM: Current and potential treatments for cervical cancer. Curr Cancer Drug Targets 13: 205-220, 2013.

27. Sánchez-Tilló E, de Barrios O, Siles L, Cuatrecasas M, Castells A and Postigo A: $\beta$-catenin/TCF4 complex induces the epithelial-to-mesenchymal transition (EMT)-activator ZEB1 to regulate tumor invasiveness. Proc Natl Acad Sci USA 108: 19204-19209, 2011.

28. Solanas G, Porta-de-la-Riva M, Agustí C, Casagolda D, Sánchez-Aguilera $F$, Larriba MJ, Pons $F$, Peiró $S$, Escrivà $M$, Muñoz A, et al: E-cadherin controls beta-catenin and NF-kappaB transcriptional activity in mesenchymal gene expression. J Cell Sci 121: 2224-2234, 2008

29. Qureshi R, Arora H and Rizvi MA: EMT in cervical cancer: Itsrole in tumour progression and response to therapy. Cancer Lett 356: 321-331, 2015

30. Kwon YJ, Ye DJ, Baek HS and Chun YJ: 7,12-Dimethylbenz[a] anthracene increases cell proliferation and invasion through induction of Wnt/ $\beta$-catenin signaling and EMT process. Environ Toxicol 33: 729-742, 2018.

31. Höckel M and Vaupel P: Tumor hypoxia: Definitions and current clinical, biologic, and molecular aspects. J Natl Cancer 93: 266-276, 2001.

32. Bos R, van der Groep P, Greijer AE, Shvarts A, Meijer S, Pinedo HM, Semenza GL, van Diest PJ and van der Wall E: Levels of hypoxia-inducible factor-1alpha independently predict prognosis in patients with lymph node negative breast carcinoma. Cancer 97: 1573-1581, 2003.

33. Birner P, Schindl M, Obermair A, Breitenecker G and Oberhuber G: Expression of hypoxia-inducible factor 1alpha in epithelial ovarian tumors: Its impact on prognosis and on response to chemotherapy. Clin Cancer Res 7: 1661-1668, 2001.

34. Song IS, Wang AG, Yoon SY, Kim JM, Kim JH, Lee DS and Kim NS: Regulation of glucose metabolism-related genes and VEGF by HIF-1alpha and HIF-1beta, but not HIF-2alpha, in gastric cancer. Exp Mol Med 41: 51-58, 2009.

35. Porta C, Paglino C and Mosca A: Targeting PI3K/Akt/mTOR signaling in cancer. Front Oncol 4: 64, 2014.

36. Bachelder RE, Yoon SO, Franci C, de Herreros AG and Mercurio AM: Glycogen synthase kinase-3 is an endogenous inhibitor of Snail transcription: Implications for the epithelial to mensenchymal transition. J Cell Biol 168: 29-33, 2005. 